\title{
Die Dokumentation des deutschsprachigen Theaters in den böhmischen Ländern durch Theateralmanache und -journale
}

Paul S. Ulrich

\begin{abstract}
Deutschsprachiges Theater fand im 19. Jahrhundert an viel mehr Orten statt, als man zunächst vermutet. Dies gilt insbesondere für geografische Gebiete außerhalb der heutigen Grenzen von Deutschland und Österreich. Entnommen einer auf Theateralmanachen und -journalen basierenden Dokumentation, die mehr als 4000 Städte umfasst (allein 427 davon in den böhmischen Ländern), wird der Band über Tschechien überraschende Einblicke in das damalige deutschsprachige Theater bieten.
\end{abstract}

\section{Schlüsselwörter}

Theateralmanache, Theaterjournale, deutschsprachiges Theater, Theater in Böhmen, Theaterspielstätten, Theaterdirektoren, Theatersouffleure

\begin{abstract}
In the 19th century, German-language theatre took place in a lot more places than was initially thought. This particularly applies to geographical areas outside the current borders of Germany and Austria. Judging by the documentation consisting of theatre almanacs and journals, which includes more than 4,000 cities (427 of them in the Bohemian lands alone), the volume on the Czech Republic will offer surprising insights into what used to be German-language theatre.
\end{abstract}

\section{Keywords}

Theatre almanacs, theatre journals, German-language theatre, theatre in Bohemia, performance venues, theatre directors, theatre prompters 
Zur Geschichte des deutschsprachigen Theaters im geografischen Gebiet der heutigen Tschechischen Republik liegen umfangreiche und bewundernswerte Arbeiten vor. ${ }^{1}$ Bislang nur teilweise berücksichtigt sind darin Theateralmanache und -journale, ${ }^{2}$ die jedoch vielfältige Informationen enthalten, die für theaterhistorische und biographische Forschungen von großem Nutzen sind und bisherige Erkenntnisse konkretisieren und erweitern können. Im Folgenden wird die Arbeit an einer umfangreichen Dokumentation des deutschsprachigen Theaters im 19. Jahrhundert vorgestellt, die eben jene Theaterperiodika als wertvolle Quellen in den Fokus nimmt: Gezeigt wird, welche Informationen in den Theateralmanachen ${ }^{3}$ stecken und welches Potenzial deren Erschließung und Aufbereitung für weitere Recherchen hat. Dies sei anhand des Beispiels zur Anzahl von Spielstätten und Orten in den böhmischen Ländern illustriert, wo deutschsprachiges Theater gespielt wurde.

Auf der Startseite des biographischen Online-Nachschlagewerks Tschechische Theaterenzyklopädie: Deutschsprachiges Schauspiel in den böhmischen Ländern im 19. Jahrhundert schreibt Berenika Zemanová Urbanová:

Im Unterschied zum tschechischen Theater, das sich in einem sprachlich und geographisch geschlossenen Raum entwickelte, erstreckt sich die deutschsprachige Theaterlandschaft über einen großen Teil des europäischen Kontinents, mit dem auch die böhmischen Länder historisch verbunden sind. Im 19. Jahrhundert waren auf dem Gebiet der heutigen Tschechischen Republik etwa 20 deutschsprachige Bühnen und eine Reihe von reisenden Theatergesellschaften tätig. ${ }^{4}$

1 Exemplarisch erwähnt seien das Nachschlagewerk (JAKUBCOVÁ und PERNERSTORFER 2013) und zwei Online-Projekte des Institut uměni - Divadelni ústav/Arts and Theatre Institute in Prag: Tschechische Theaterenzyklopädie: Deutschsprachiges Schauspiel in den böhmischen Ländern im 19. Jahrhundert [online], Praha, (http://de.encyklopedie.idu.cz/index.php/Kategorie:Deutschsprachiges_Schauspiel) und die OnlineDatenbank Europäische Theaterarchitektur/European Theatre Architecture (EUTA) (https://www.theatrearchitecture.eu/de/).

2 Vgl. (HAVLÍČKOVÁ, PRACNÁ und ŠTEFANIDES 2011-2014). Besonders im zweiten und im dritten Band wird auf Einträge zu den einzelnen Orten in Almanachen verwiesen, ebenso wie auf das Vorhandensein von Theaterjournalen zu diesen Orten, mit Hinweisen zu den Fundstellen.

3 Unabhängig von den sehr unterschiedlich verwendeten Titeln der Theaterperiodika wird generell zwischen universalen Almanachen und lokalen Journalen unterschieden.

Universale Almanache behandeln mehr als eine Gesellschaft oder Spielstätte und wurden als jährliche Publikation konzipiert, d.h. sie sind fortlaufende Publikationen. In der Regel schickten die Direktoren zu Beginn der Saison Angaben über ihre Gesellschaft an die Redaktion und die Almanache erschienen um Weihnachten. Da sie über die normalen Buchvertriebswege verkauft wurden, war die Anzahl der gedruckten Exemplare groß, weshalb viele von ihnen erhalten geblieben sind.

Lokale Journale befassen sich nur mit einer Gesellschaft oder einer Spielstätte. Mit Ausnahme einiger Journale von Hoftheatern wurde jeweils nur eine geringe Anzahl von Exemplaren gedruckt und sie waren nur in der Stadt erhältlich, in der die Theatergesellschaft auftrat. Bislang konnten mehr als 6.000 Einzeltitel für das deutschsprachige Theater nachgewiesen werden. Wenn man die Anzahl der in den Almanachen genannten Orte als Maßstab nimmt und den Zeitraum von über einem Jahrhundert zu Grunde legt, lässt sich hochrechnen, dass mehr als 100.000 Journale produziert worden und somit noch zu entdecken sind.

4 Verfügbar online http://de.encyklopedie.idu.cz/index.php/Kategorie:Deutschsprachiges_Schauspiel. 
Die Zahl 20 dürfte sich auf explizite Theater-Gebäude beziehen - womit jedoch außer Acht gelassen wird, dass in vielen Orten auch Restaurants oder Hotels als Veranstaltungsorte für Theater genutzt wurden. ${ }^{5}$ Weitere Begriffe, mit denen Spielstätten bezeichnet wurden, etwa „Blinden-Anstalt“, „Braukommune“, „Glassalon“, „Herrenhaus“, „Schießstatt“, „Schützenhaus“, „Turnhalle“ oder „Vereinshalle“, können ebenso verwirren und eine Identifikation als Bühne erschweren.

Wie die hier vorgestellte Dokumentation zeigt, war die Anzahl sowohl der Spielstätten als auch der mobilen Theater-Gesellschaften im Gebiet der heutigen Tschechischen Republik deutlich höher als die genannte Zahl 20. Die Herausforderung besteht in der Suche nach und Identifizierung von Gesellschaften und Spielstätten, und hierfür sind universale Theateralmanache mit ihren Informationen zu Spielstätten und Gastspielen ein erfolgsversprechender Ausgangspunkt, denn in der Auswertung für Tschechien werden über 700 Spielstätten genannt. ${ }^{6}$

Die universalen Almanache geben Auskunft über die Bedingungen, unter denen das deutschsprachige Theater funktionierte, und sie machen die Mobilität von Theatergesellschaften und deren Notwendigkeit verständlich: Mobilität war (und bleibt) ein wichtiger Faktor im Theater, die meisten Gesellschaften blieben selten länger als ein paar Saisonen an einem bestimmten Ort. ${ }^{7}$ Während des größten Teils des 19. Jahrhunderts hatten die meisten Orte unter 30.000 Einwohner und die Spielstätten verfügten im Schnitt über zwischen 400 und 800 Sitzplätze. ${ }^{8}$ Diese Rahmenbedingungen und ihre wirtschaftliche Implikationen prägten die Spielplan-Erstellung: Stücke konnten nicht en suite gespielt werden, stattdessen gab es tägliche Stückwechsel. Strategien der Theaterdirektoren, mit den Beschränkungen umzugehen, bestanden unter anderem darin, entweder hintereinander oder parallel in benachbarten Orten aufzutreten. ${ }^{9}$ Ob eine Gesellschaft einen oder mehrere Sitze für ihre Tätigkeit hatte oder ob es sich um ambulante Unternehmen ohne festen Sitz handelte, ist im Nachhinein nicht immer eindeutig feststellbar. Die Situation wird dadurch verkompliziert,

5 Dass Spielstätten in Restaurants und Hotels oft „Stadttheater“ genannt wurden, kann zur Annahme verleiten, dass es sich um ein permanentes Gebäude handle; tatsächlich wird damit eine Theater-Gesellschaft mit einer Konzession für den jeweiligen Ort bezeichnet, nicht ein Gebäude.

6 Diese Zahl berücksichtigt, dass Spielstätten teilweise mehrere Benennungen hatten, da es im Laufe der Zeit nicht ungewöhnlich war, dass sich aufgrund neuer Besitzer bzw. Theaterdirektoren die Namen der Spielstätten änderten. Dafür sind die mindestens zweimal so vielen unbenannten Spielstätten der ambulanten Gesellschaften nicht berücksichtigt.

7 Eine Abweichung besteht vielleicht in der Zusammensetzung der ursprünglichen Hoftheater. Aber auch hier kam es regelmäßig zu Fluktuationen innerhalb der Gesellschaft.

8 Interessant bei der Erstellung der Dokumentation war die Beobachtung, dass in den Städten im nichtdeutschsprachigen Raum die Einwohnerzahl in der Regel höher war als in vergleichbar großen Städten in Deutschland und Österreich, und dass auch der Anteil der deutschsprachigen Bevölkerung oft größer war als jener in deutschen und österreichischen Städten. Dies gilt nicht nur für die Tschechische Republik und Polen, sondern vor allem für die großen Städte der Vereinigten Staaten von Amerika, wo der Anteil der Bevölkerung mit deutschen Wurzeln zum Teil die Einwohnerzahl von Berlin und Wien überstieg.

9 Diese Art der Mobilität stellt für die Erarbeitung einer Dokumentation ein Problem dar, da in den Almanachen in der Regel jener Ort als Hauptsitz der Gesellschaft angenommen wurde, von dem aus die Informationen an die Redaktion gesandt wurden, und dies variiert von Almanach zu Almanach, von Jahr zu Jahr. 
dass es Gesellschaften gab, die an einem Ort ansässig waren und zusätzlich Gastspiele in anderen Orten gaben - und dass die Almanache diesen Umstand nicht immer gleich behandelten. ${ }^{10}$

Abhängig davon, wie die Information in den Almanachen präsentiert ist, werden die Unternehmen in der Dokumentation entweder als in einer Stadt ansässig aufgeführt, oder es wird in Fußnoten ergänzt, wenn sie in der vergangenen Saison in dieser Stadt gastierten.

Die topografischen Informationen, die die Dokumentation aus den universalen Almanachen extrahiert, bringen teils überraschende Erkenntnisse: Sowohl auf dem Gebiet der heutigen Tschechischen Republik als auch auf dem Gebiet des heutigen Polen sind mehr Orte mit Theateraktivitäten dokumentiert (je 427) als im Gebiet des heutigen Österreich (320). Allerdings ist zu bedenken, dass diese Zahlen keineswegs die gesamte Präsenz des deutschsprachigen Theaters in diesen Regionen widerspiegeln, da ambulante Gesellschaften erst gegen Ende des 19. Jahrhunderts in den Almanachen aufgeführt werden. Zu diesem Zeitpunkt ist die Zahl der dokumentierten reisenden Gesellschaften sehr hoch, und es ist davon auszugehen, dass sie mindestens ebenso aktiv waren, bevor sie in Theaterperiodika erfasst wurden; damit wäre man berechtigt zu vermuten, dass die Anzahl der Städte, in denen Aufführungen stattfanden, wesentlich größer ist. ${ }^{11}$

Der Zeitraum, den die Dokumentation abdeckt, erstreckt sich über mehr als 100 Jahre, und das Theater verändert sich konstant: Im Laufe eines Jahrhunderts brennen Gebäude ab und werden entweder wiederaufgebaut oder es wird ein gänzlich neuer Bau am selben oder an einem anderen Ort errichtet. Die Eigentumsverhältnisse an den Gebäuden wechseln und damit einher geht oft die Vergabe eines neuen Namens für die (neue) Spielstätte - anhand von Beispielen wie diesen werden die Herausforderungen bei der Erstellung einer Dokumentation greifbar. Anstatt zu versuchen, über Vereinheitlichungen zu simplifizieren (und mit derartigen Glättungen zu riskieren, historische Realitäten zu verwischen), wird der Darstellung zum Zeitpunkt des Erscheinens des jeweiligen Almanachs gefolgt; geringfügige Namensschwankungen werden etwa durch die Verwendung von Querverweisen angezeigt.

Wie schon allein die Anzahl der Orte, in denen Almanache deutschsprachiges Theater dokumentieren, nicht genau sein kann, so ist die Anzahl der Spielstätten noch schwieriger zu bestimmen. Dies ist auf verschiedene Faktoren zurückzuführen: Erstens gibt es keine Übereinstimmung und Konsistenz in dem, was mit den Organisationen gemeint ist, die in den Einträgen aufgeführt werden: In vielen Fällen

10 Die Darstellung variierte wiederum von Almanach zu Almanach und von Jahr zu Jahr. In einigen Fällen lässt sich dies dadurch erklären, dass eine Gesellschaft eine Stadt besucht hat und mindestens eine weitere Saison in dieser Stadt blieb, weil sie gut aufgenommen wurde.

11 Nicht zu vergessen ist, dass die ambulanten Gesellschaften alle möglichen Räumlichkeiten für ihre Aufführungen benutzt haben. Sofern kein geeigneter Spielort vorhanden war, wurde - wie Fr. Glindmeier in einer Mitteilung in Die Heimat, Jg. 18 (1908), H. 7, S. 181 beschrieben hat - sogar in Scheunen im Beisein von Vieh gespielt. 
handelt es sich um eine organisatorische Einheit, die an mehreren Spielstätten aufgetreten ist, ${ }^{12}$ sie kann sich aber auch auf ein Gebäude beziehen, in dem mehrere Gesellschaften aufgetreten sind. ${ }^{13}$ Zweitens fehlt in den Almanachen bei der Aufzeichnung beinahe jeden Ortes die Angabe der Veranstaltungsorte, in denen Unternehmen gastierten. Drittens kommen unterschiedliche Bezeichnungen für die jeweilige Spielstätte hinzu, was insbesondere für Kurorte gilt, wo etwa auf ein oder mehrere Hotels mit Zusatzbezeichnung als Kurtheater verwiesen wird, oder in anderen Einträgen nur die Bezeichnung „Kurtheater“ verwendet wird. Ähnlich verhält es sich mit der Benennung eines Veranstaltungsortes als "Stadttheater“ in dem einen Almanach und als „Restaurant" oder „Hotel“ in einem anderen. Fanden Veranstaltungen im Sommer statt, gab es neben Aufführungen in einem Gebäude oft auch welche in einem Freilufttheater.

Obwohl die Zahl der tschechischen Orte, in denen deutschsprachiges Theater präsent war, groß ist, ist die Anzahl der bislang lokalisierten lokalen Theaterjournale - mit Ausnahme einiger weniger Städte wie insbesondere Prag und Olmütz - relativ gering. Dies kann daran liegen, dass vorhandene Exemplare bislang nicht katalogisiert wurden oder dass Institutionen, die sie besitzen, nicht über leicht zugängliche Recherchewerkzeuge wie OPACs verfügen, um sie zu finden. ${ }^{14}$ Ebenfalls denkbar ist, dass veröffentlichte Journale nicht aufbewahrt oder gesammelt wurden.

Das im Folgenden vorgestellte Projekt ist auf Vollständigkeit angelegt. Mit der Veröffentlichung der regionalen Auszüge der Dokumentation wird - wie auch mit diesem Aufsatz - gleichzeitig ein Appell an Forscher und besitzende Einrichtungen gerichtet, bislang nicht verzeichnete vorhandene (weitere) Theaterjournale ${ }^{15}$ an den Autor bzw. an das Don Juan Archiv Wien zu melden, um die Bibliografie der Theaterjournale zu ergänzen.

12 Ein Paradebeispiel hierfür ist das Berliner Hoftheater, das den Namen „Königliche Schauspiele“ trug. Neben dem Königlichen Schauspielhaus und dem Königlichen Opernhaus in Berlin trat das Ensemble auch im Königlichen Schauspielhaus in Potsdam und im Königlichen Schloßtheater in Charlottenburg auf; zudem trat das Ensemble zu bestimmten Zeiten in anderen Spielstätten in Berlin auf.

13 Dies kann der Fall sein, wenn ein Theaterdirektor eine Konzession für die Wintersaison und eine andere Konzession für die Sommersaison hatte.

14 Dies ist beunruhigend, da die ungarische Bibliographie von Theaterkalendern von (HANKISS und BERCZELI 1961) zeigt, dass es nicht nur viele deutschsprachige Theaterjournale in Ungarn und Rumänien gab, sondern dass auch sehr viele ungarische Journale gedruckt wurden. Auf Tschechien übertragen würde dies bedeuten, dass bislang offensichtlich keine größeren Anstrengungen unternommen wurden, um ihre Präsenz zu dokumentieren.

15 Der aktuelle Stand zu einem Ort oder einer besitzenden Einrichtung kann im OPAC www. theaterjournale.at abgerufen werden. 


\section{Das Projekt ${ }^{16}$}

Theateralmanache und -journale des deutschsprachigen Theaters stehen im Fokus eines Gemeinschaftsprojektes des Don Juan Archivs Wien und des Autors. Die darin gesteckten Ziele sind die Digitalisierung der Sammlung an universalen Almanachen und lokalen Theater-Journalen, die der Autor in den letzten 40 Jahren aufgespürt hat, das Zugänglichmachen der formal erfassten Titel- und Nachweisdaten sowie die Publikation von deren inhaltlicher Erschließung und Auswertung. In den vier Jahren seit dem Projektstart hat sich viel getan und über den Verlauf des Projektes und das Erreichen von Meilensteinen wurde in Aufsätzen (PERNERSTORFER und GRUBER 2015; PERNERSTORFER 2018), auf Tagungen ${ }^{17}$ und über die Website des Don Juan Archivs ${ }^{18}$ berichtet: Mit Theaterpublizistik 1750-1918 digital ist ein umfangreiches digitales Archiv erstellt worden, das aktuell über 4.000 Theaterperiodika-Digitalisate umfasst und kontinuierlich wächst, ${ }^{19}$ und die bibliographischen Informationen zur Sammlung samt Besitzvermerken wurden in einem Online-Katalog zugänglich gemacht, ${ }^{20}$ in dem die in knapp 400 Einrichtungen weltweit nachgewiesenen Journale und Almanache institutionsübergreifend recherchiert werden können. Dieser Beitrag möchte nun einen Einblick geben in die Umsetzung des dritten Zieles: die Dokumentation des deutschsprachigen Theaters bis $1918^{21}$ durch die Publikation der inhaltlichen Auswertungen der Almanache und Journale.

Diese detaillierte Bibliographie erscheint als mehrbändige Reihe Topographie und Repertoire des Theaters, deren erster Band zum Theater in Wien im Jahr 2018 veröffentlicht wurde. ${ }^{22}$ Weitere Bände sind in Arbeit und können vor ihrer Veröffentlichung bereits teilweise als Vorabdrucke im Don Juan Archiv eingesehen werden, unter anderem der Band zum Theater in Tschechien. ${ }^{23}$

16 Die nun folgenden Ausführungen zeichnen die Entstehung des Projekts anhand des Wiener Theaters nach und wurden mit kleineren Abweichungen auch in (ULRICH 2019) veröffentlicht.

17 Beispielsweise bei den 42. Internationalen Nestroy-Gesprächen 2016 in Schwechat, bei den Tagungen „Digitale Bibliothek“ 2016 in Graz, „Theorie und Praxis im Theater im 17-19. Jahrhundert“ 2018 in Eger (Ungarn) oder auf der Konferenz „Digital \& Archival Approaches to Theater History“ im Januar 2019 in Philadelphia.

18 Verfügbar online http://www.donjuanarchiv.at/vorlaesse-leihgaben/slg-paul-s-ulrich.html.

19 Zugänglich ist das digitale Archiv im Don Juan Archiv Wien (Trautsongasse 6, 1080 Wien, www. donjuanarchiv.at) sowie beim Autor in Berlin.

20 Verfügbar online http://www.theaterjournale.at/.

21 Das Jahr 1918 wurde deshalb als Abgrenzung gewählt, weil nach dieser Zeit große Umstrukturierungen des Theaters stattfanden - im Gegensatz zu den bisher privat geführten Organisationen wurden die Theater nun zunehmend von den Kommunen getragen. Nach 1918 wurden zudem keine (privaten) Souffleur-Journale veröffentlicht; wenn Almanache veröffentlicht wurden, waren es Veröffentlichungen des Theaters.

22 Vgl. (ULRICH 2018).

23 Neben Tschechien sind gegenwärtig als Vorabdrucke vorhanden: Südosteuropa (Bosnien und Herzegowina, Bulgarien, Georgien, Griechenland, Kroatien, Rumänien, Serbien, Slowakei, Slowenien, Türkei, Ukraine, Ungarn), Amerika (Argentinien, Brasilien, Chile, Jamaika, Kanada, Kuba, Mexiko, Peru, Uruguay, USA) und Nordeuropa (Dänemark, Estland, Finnland, Lettland, Litauen, Norwegen, Schweden, Russland, Weißrussland). 
Bei der Vorbereitung des Materials über das deutschsprachige Theater auf dem Gebiet der Republik Tschechien haben Kollegen in Tschechien großartige Unterstützung geleistet. In der Frühphase des Projektes hat das Institut umění - Divadelní ústav in Prag durch die Identifizierung und Zurverfügungstellung von Kopien tschechischer Theaterjournale große Hilfe geleistet. Der Dank des Autors gilt hier insbesondere Alena Jakubcová und Adolf Scherl (1925-2017). Kolleginnen und Kollegen aus Tschechien haben durch ihre aufmerksame Sichtung des Vorabdruckes des Tschechien-Bandes und viele wertvolle Hinweise und Vorschläge geholfen. Insbesondere die Expertise von Jiř́ Š Stefanides und Miroslav Lukáš war bei der Überprüfung der oft verwirrenden Städtenamen in den Almanachen unverzichtbar, sie haben zudem die Suche nach und die Beschaffung von Kopien unterstützt.

Die regionalen Vorabdrucke dokumentieren und prägen gleichzeitig das Wachsen und Werden der Bibliographie, denn durch die Herausforderungen bei der Erstellung der einzelnen Bände und der jeweiligen regionalen Spezifika entwickelt sich eine eigene Dynamik, Kurskorrekturen eröffnen neue Perspektiven der Herangehensweise und führen zu inhaltlichen Erweiterungen, die umso eindrücklicher deutlich machen, welcher Informationsschatz in diesen theaterpublizistischen Quellen liegt, den es für vielfältigste Forschungsfragen zu heben gilt. Dieser Entwicklungsprozess soll im Folgenden skizziert werden.

\section{Vom Plan einer ,simplen' Bibliographie ...}

Der ursprüngliche Plan war schlichtweg, die Materialien beider Publikationsarten lokale Journale und universale Almanache - in einer einzigen Bibliographie zu präsentieren. Rasch stellte sich heraus, dass dies ohne Einbußen hinsichtlich Übersichtlichkeit schwer realisierbar ist, da Inhalt und Zielgruppe zu unterschiedlich sind. Die Journale waren eine Art Souvenir für das Publikum, wohingegen die Almanache als Fachpublikationen für Theaterdirektoren, Schauspieler und Regisseure konzipiert waren. Aus pragmatischer Sicht kam hinzu, dass für eine konsistente Behandlung der lokalen Journale weitere umfangreiche Recherchen notwendig waren, da bislang nur etwa zwei Drittel der bekannten Publikationen zugänglich waren und eine Autopsie durchgeführt werden konnte; zudem werden - vor allem auch durch die Arbeit an der Bibliographie - laufend neue Journale identifiziert, deren Einarbeitung neue Informationen bringt. Auch aus strategischen Gründen erschien es daher sinnvoller, die beiden Publikationsarten als Grundlage gedanklich zu trennen und sich zunächst auf die universalen Almanache zu konzentrieren: Diese sind fast vollständig verfügbar und auch die Indexierung ist weitgehend abgeschlossen.

In Vorbereitung: Polen, Berlin, Westeuropa (Belgien, England, Frankreich, Irland, Liechtenstein, Luxemburg, Niederlande, Schottland, Wales), Südeuropa (Gibraltar, Italien, Malta, Monaco, Portugal, Spanien, Schweiz), Österreich und die einzelnen deutschen Bundesländer. Wie Wien unterscheidet sich Berlin von den meisten Städten; es gab in Berlin nicht nur viel mehr Spielstätten (nach 1871 mehr als 50), sondern auch viele in Berlin ansässige Tourneeveranstalter, und schließlich war es die Stadt mit den meisten Zeitungen. Im Gegensatz zu Wien, der Stadt mit den meisten lokalen Journalen, gab es in Berlin davon nur sehr wenige. 
Die umfassend angelegte Indexierung, also vollinhaltliche Erschließung der Almanache wurde aus praktischen Grunde in zwei Teilen vorgenommen: Die Teile, die der Beschreibung der einzelnen Theater gewidmet sind, wurden von den mehr journalistisch angelegten Teilen getrennt und als erste Unternehmung veröffentlicht. Dies eröffnete wiederum die Möglichkeit, sich bei der Wiedergabe der einzelnen Spielstätten bzw. Gesellschaften, auf die sich der Hauptteil der universalen Almanache bezieht und die auch im Mittelpunkt der Dokumentation stehen, nicht nur wie ursprünglich geplant auf die Präsentation der Namen der Veranstaltungsorte mit den Direktoren samt Quellenverweis zu beschränken, sondern darüber hinauszugehen: Die Almanache enthalten oft weitere Informationen über die Städte (historische Informationen, Bevölkerungszahl etc.) und zu den Spielstätten (Baubeginn, Anzahl der Sitzplätze, weitere von der Gesellschaft besuchte Städte, Gäste etc.). Die Entscheidung für die Aufnahme der ortspezifischen Informationen aus den Almanachen legten eine weitere, sozusagen fachspezifische Ergänzung um Ortsbeschreibungen aus zeitgenössischen Theaterlexi$\mathrm{ka}^{24}$ nahe.

An dieser Stelle sei erwähnt, dass abgesehen von diesen Ortsbeschreibungen aus den Enzyklopädien für die Dokumentation lediglich die Informationen aus den aufgeführten Theaterpublizistik-Quellen verwendet werden, mit kleineren, gekennzeichneten Abweichungen: Größere orthographische Variationen wurden in den meisten Fällen mit Querverweisen beibehalten; ${ }^{25}$ die Namen und Lebensdaten der Direktoren, Souffleure und Kritiker wurden entweder in Übereinstimmung mit den Einträgen in meinem Biographischen Verzeichnis (ULRICH 1997) oder jenen in anderen biografischen Quellen angepasst. Die Namensgebung der Orte in den Almanachen war oft problematisch.

Auch hinsichtlich der geplanten Struktur der Präsentation ergab die konkrete Arbeit mit dem Material Änderungen bezüglich der ursprünglichen Überlegung, alphabetisch nach Städten zu sortieren, gefolgt von den eben erwähnten allgemeinen Informationen über die jeweilige Stadt und einer alphabetischen Auflistung der Veranstaltungsorte, mit jeweils einer chronologischen Auflistung (nach dem Datum der Veröffentlichung) der Quellen. Obwohl dies im Gedanken unkompliziert erscheint, zeigte sich bei der Vorbereitung der Einträge, dass die Angaben in zwei oder mehr parallel erschienenen Almanachen deutlich voneinander abweichen können, sodass für jedes Jahr mehrere Einträge mit unterschiedlichen und oft widersprüchlichen Informationen vorhanden sein können. ${ }^{26}$

24 Übernommen werden die Ortsbeschreibungen aus den folgenden Almanachen: (BLUM, HERLOSSSOHN und MARGGRAFF 1839-1846; SACHSE 1853-1872; OPPENHEIM und GETTKE 1889).

25 Es gab jedoch Ausnahmen, insbesondere bei den mit und ohne Bindestrich versehenen Namen von Theatern, die standardisiert wurden, zum Beispiel „Stadttheater“ für die Schreibweisen „Stadt-Theater“ und „Stadtheater“. Für unterschiedliche Ortsnamen ist angestrebt, die im 19. Jahrhundert „offizielle bzw. amtliche“ Schreibweise zu verwenden; daher „Cöln“ statt „Köln“ etc.

26 Je nachdem, wann die Informationen an die Almanach-Redaktion geschickt wurden, kann es zu einer Änderung des Namens oder einer abweichenden Bezeichnung des Veranstaltungsortes gekommen sein; es kann auch eine Änderung der Leitung stattgefunden haben oder veraltetes Material aus dem vorherigen Band wurde auf den neuen Band übertragen, weil aktuelle Informationen nicht zur Verfügung gestellt 
Statistische Auswertungen der Daten ergaben, dass von den mehr als 4.000 erwähnten Orten einige mit nur einem Eintrag in einem Almanach verzeichnet sind, während bei anderen zahlreiche Spielstätten über den gesamten Zeitraum, also von 1752 bis 1918, genannt werden. Wie dieses Material am besten aufbereitet werden konnte, war eine offene Frage, die prädestiniert ist, um sie anhand einer konkreten Stadt zu klären. Die Auswahl dafür fiel nicht schwer: Da sich das Don Juan Archiv in Wien befand und zudem die meisten Journale in Wien produziert wurden, war das Feld des Pilotprojekts schnell gefunden.

\section{... über ein Pilotprojekt ...}

Wien also. Eine Liste zu den Wiener Journalen aus früheren Auswertungen wurde die Basis einer zu diesem Zeitpunkt noch als ,Broschüre' konzipierten bibliographischen Sammlung, deren Umfang sich im Folgenden radikal ausdehnen sollte. Bei der konkreten Arbeit an den Wiener Daten offenbarten sich weitere Herausforderungen, die zu zahlreichen Änderungen führten. Das betrifft im topographischen Kontext etwa Orte im Umkreis von Wien, die erst im 20. Jahrhundert in Wien eingemeindet wurden, davor jedoch eigenständige Gemeinden waren; sie werden als „Wiener Vororte“ getrennt angeführt.

Im Bereich der Mobilität wurde für in Wien ansässige Tournee-Unternehmen ein eigener Bereich geschaffen, um auch diese Informationen kompakt zur Verfügung zu stellen. Eine weitere wesentliche Ergänzung stellen die Itinerare der Direktoren dar: Die Tätigkeit der Direktoren war nicht auf Wien beschränkt, sie konnte eine oder mehrere der über 4.000 Städte umfassen - die Informationen hierzu stammen aus dem Datenpool der Auswertung aller Almanache. Damit wird nicht nur deutlich, wie mobil die Theaterdirektoren waren, ${ }^{27}$ sondern auch Ausblick darauf gegeben, wie wertvoll die endgültige Gesamtdokumentation sein wird.

Eine Besonderheit von Wien ist, dass es Wiener Journal-Veröffentlichungen gibt, die mehrere Theater umfassen und damit zu den universalen Almanachen zählen; diese Einstufung hat interessante Konsequenzen für Struktur und Inhalt der Bibliographie, da sie einen Weg eröffnete, Journale teilweise in die Dokumentation der Almanache zu integrieren.

Anstatt die Wiener Journale lediglich alphabetisch aufzulisten, wurde ein anderer Ansatz gewählt: Da viele der Journale Repertoire-Verzeichnisse beinhalten, bot eine chronologische Auflistung der Repertorien mit ihrer Zuordnung zu einem Journal

wurden - dies gilt insbesondere für Angaben über Bevölkerungszahl eines Ortes und Anzahl der Plätze in einer Spielstätte.

27 So aufschlussreich die Reiserouten der Wiener Theaterdirektoren auch sind, die Mobilität ist in den nachfolgenden regionalen Vordrucken viel stärker zu erkennen. Ein Vergleich des Umfangs der Itinerare der Theaterdirektoren im Wien-Band mit den Tschechien- und Südosteuropa-Bänden verdeutlicht dies. Im Wien-Band umfassen die Itinerare 60 Seiten, im Tschechien-Band sind es 105 Seiten und im SüdosteuropaBand 100 Seiten. 
oder einem Almanach ${ }^{28}$ einen neuen Zugang zu bestimmten Fragestellungen, die von Journalen beantwortet werden können. Eine separate Auflistung von Journalen, die entweder noch nicht eingesehen werden konnten, oder aber kein Repertoire enthalten, informiert über die zusätzlich bekannten Wiener Journale. Mit der speziellen Kennzeichnung der bislang nicht zugänglichen Journale wird auch der Hoffnung Rechnung getragen, dass Forschende oder besitzende Einrichtungen den Autor oder das Don Juan Archiv über vorhandene Bestände und auch neue Funde bislang unbekannter Journale informieren.

Eine weitere Ergänzung war die Auflistung der Souffleure mit den Titeln der von ihnen veröffentlichten Wiener Journale. ${ }^{29}$ Insbesondere in der zweiten Hälfte des 19. Jahrhunderts begannen die Redaktionen in den Almanachen nicht nur Zeitungen zu nennen, die über das Theater berichteten, sondern auch die Namen und Adressen der Kritiker bekannt zu geben. Da auch diese Informationen für die Forschung von großer Bedeutung sein können, wurden dazu zwei neue Abschnitte in die ,Broschüre' aufgenommen, die auf über 350 Seiten angewachsen war - und somit war auch klar, dass eine vollständige Dokumentation nur in Form eines mehrbändigen Werkes realisierbar ist.

\section{... zur Struktur der Bibliographie}

Anhand des Wien-Bandes konnte ein Modell entwickelt werden, das die Informationsfülle des verfügbaren und erschlossenen Materials in eine Struktur bringt, die es für unterschiedlichste Forschungsfragen handhabbar macht:

\section{TOPOGRAPHISCHE GESICHTSPUNKTE}

Spielstätten in Wien. Der größte Abschnitt widmet sich den Spielstätten in Wien, alphabetisch sortiert: in chronologischer Reihenfolge werden die Direktoren aufgeführt, samt Angabe des Almanachs, aus dem die Information stammt. Die Almanache verzeichnen in der Regel die gesamte Gesellschaft, ${ }^{30}$ oft mit den Adressen in Wien und häufig mit zusätzlichen

28 Gelegentlich enthielten die universalen Almanache auch Repertoireverzeichnisse einzelner Theater dies gilt insbesondere für den in Berlin erschienenen Almanach für Freunde der Schauspielkunst von Ludwig Wolff, der zuerst Souffleur war und danach Theateragent, und das Repertoire der Königlichen Schauspiele erfasste.

29 Die Auflistung der Namen von Souffleuren kann hilfreich sein beim Auffinden von Journalen, deren mitunter kreative Titel nicht immer auf den ersten Blick vermuten lassen, dass es sich um ein Theater-Journal handelt.

30 Der Autor hat seine Datenbank, aus der sich seine biographischen Verzeichnisse (etwa Biographisches Verzeichnis für Theater, Tanz und Musik) speisen, um Informationen über die Mitglieder der Gesellschaften aus den Almanachen erweitert und neben den Namen auch Informationen darüber erfasst, wann und wohin sie die Gesellschaft verlassen haben, sowie alle Gastspiele von Einzelpersonen. Mithilfe dieser Datenbank ist es nunmehr möglich, die beruflichen Karrieren aller am deutschen Theater beteiligten Personen zu verfolgen. Die Universitätsbibliothek Frankfurt am Main ist derzeit dabei, diese Datenbank (d.h. sowohl die biographischen Verzeichnisse als auch die Informationen über die Mitglieder der Gesellschaften) online verfügbar zu machen. Wann diese Migration abgeschlossen sein wird, ist noch ungewiss. 
Informationen, beispielsweise zu den neuen Stücken im Repertoire. Wenn spezifische Informationen über den Veranstaltungsort gegeben werden (z.B. wann das Theater gebaut oder umgebaut wurde, Anzahl der Plätze, wann Aufführungen stattfanden, Aspekte der Behandlung der Schauspieler oder etwaigen Ruhestands- oder Unfallkonzepten), werden diese in Fußnoten wiedergegeben. ${ }^{31}$ Ebenfalls in den Fußnoten enthalten sind Informationen über Gastspiele von auswärtigen Gesellschaften in der jeweiligen Spielstätte. ${ }^{32}$

Spielstätte in den Wiener Vororten. Diese Orte wurden inzwischen in das heutige Wien integriert.

\section{MOBILITÄT}

Tourneetruppen mit Sitz in Wien. Hier werden Gastspielgruppen und Unternehmen verzeichnet, die Wien zugeordnet werden können. ${ }^{33}$

Gesellschaften, die in Wien gastierten. In diesem Abschnitt wurden jene Gesellschaften genannt, die in Wien auftraten, für die jedoch kein Veranstaltungsort angegeben wurde. ${ }^{34}$

Itinerare der Direktoren. Hier sind alle Direktoren der Theater in Wien aufgeführt. Neben ihrer Tätigkeit in Wien sind auch alle Theater aus der gesamten Dokumentation aufgeführt. ${ }^{35}$

\section{REPERTOIRE}

Theater-Journale ohne Repertoire. Nicht alle Wiener Journale enthielten Angaben zum Repertoire. Unterschieden wird in diesem Abschnitt zwischen Journalen ohne Repertoire und den Titeln derjenigen Journale, die zwar bibliographisch identifiziert, aber zum Zeitpunkt des Drucks noch nicht geprüft werden konnten.

Theater-Journale mit Spielplan. Wenn in Journalen und Almanachen Angaben zum Repertoire enthalten sind, werden diese in chronologischer Reihenfolge angeführt, mit konkretem Zeitraum, Spielstätte und Quellenangaben. ${ }^{36}$

Souffleure und andere Personen, die Wiener Journale herausgaben. In diesem Abschnitt sind die Personen (in der Regel war es der Souffleur) aufgeführt, mit einer Auflistung der von ihnen veröffentlichten Wiener Journale.

31 Im Band Wiener Theater (1752-1918) gibt es 1119 Fußnoten in diesem Abschnitt.

32 Nicht enthalten sind Gastspiele von Personen, da diese Informationen in der Datenbank enthalten sind, die ins Internet migriert werden soll (siehe Fußnote 23).

33 Diese Informationen beziehen sich ausschließlich auf Angaben, die unter der Überschrift „Wien“ in den Almanachen zu finden waren. Enthalten ist keine Information über die Gesellschaften, die in den Städten außerhalb Wiens gelistet sind, da zum Zeitpunkt der Veröffentlichung des Wien-Bandes nicht alle Informationen aus allen anderen Städten überprüft worden waren. Sie werden in einer späteren Ausgabe des Wiener-Bandes und auch in der endgültigen Dokumentation enthalten sein.

34 Die Situation ist hier ähnlich wie im vorherigen Abschnitt, d.h. es werden in einer späteren Ausgabe und in der Gesamt-Dokumentation mehr Unternehmen aufgeführt werden.

35 Das volle Ausmaß dieser Mobilität zeigt sich nur teilweise im Wien-Band, da sich Wien an der Spitze der sog. „Theaterpyramide“ befand und die Theaterdirektoren dazu neigten, viel länger in Wien zu bleiben als an anderen Orten.

36 Die Informationen aus den Journalen sind minimal. Der vollständige Inhalt der Journale wird in der Gesamtbibliographie der lokalen Theater-Journale verfügbar sein. 


\section{ZEITUNGEN, REDAKTEURE UND KRITIKER}

Wiener Zeitungen, die in den Almanachen aufgeführt sind. Besonders ab Mitte des 19. Jahrhunderts begannen die Almanache, jene Zeitungen aufzulisten, die über das Theater berichteten. Die Liste der Zeitungen ist alphabetisch mit chronologischen Quellenangaben. Öfters ist auch aufgeführt, wo sich die Redaktionen befanden, und zusätzlich zu den Namen von Redakteur und Kritiker waren auch Informationen zu finden, wo der Kritiker wohnte bzw. wo sein Büro war.

Redakteure und Kritiker in Wiener Zeitungen. In diesem Abschnitt sind die Redakteure und Kritiker mit Informationen darüber aufgeführt, für welche Zeitung(en) sie wann gearbeitet haben - gemäß den Angaben in den Almanachen.

\section{Neufokussierung}

Bei der Arbeit an den Bänden für die Region Südosteuropa und für Tschechien verlagerte sich der Fokus von einer mehr oder weniger kompakten Großstadt auf ein ganzes Land bzw. über Ländergrenzen hinweg auf eine ganze Region. In dieser veränderten Blickweite offenbarte sich, dass durch die Ergänzung der Dokumentation um weitere Abschnitte neue Fragestellungen beantwortet werden können:

- Unternehmen mit Konzessionen für eine Region. Im Ortsverzeichnis der regionalen Bände sind die Gesellschaften einer bestimmten Stadt zugewiesen, allerdings ist schwer zu erkennen, welche von ihnen wirklich ambulant und in welchem geografischen Gebiet sie tätig waren. Die Auflistung des Gebiets, für das die Gesellschaft eine Konzession hatte, lässt erkennen, dass die genannten Städte wahrscheinlich nur ein Bruchteil derjenigen bilden, die besucht wurden, und dass es vorteilhaft wäre, auch andere Städte in dieser Region als mögliche Spielorte zu berücksichtigen. ${ }^{37}$

- Itinerare von Souffleuren in den Almanachen. Diese Informationen stammen aus Personalverzeichnissen in denjenigen Almanachen, die vom Verfasser in seiner Datenbank bis jetzt erfasst wurden, in der Hoffnung, dass aufgrund dieser Informationen theaterhistorisch Forschende weitere, bislang noch nicht erfasste Veröffentlichungen dieser Souffleure identifizieren und das Don Juan Archiv über derartige Neuentdeckungen informieren, um die Bibliographie der lokalen Journale zu vollständigen. Hinweise auf diejenigen Souffleure, welche Titel im Abschnitt Journale haben, werden gegeben.

- Direktoren der Gesellschaften mit Journalen. Neben Jahr und Titel der Journale sind auch die Daten der Repertoires aufgeführt. Ähnlich wie die Behandlung der Souffleure in den Almanachen werden auch die Einträge zu den Itineraren der Direktoren einen Hinweis darauf enthalten, dass es für diese Direktoren Journale gibt.

37 Die regionale Zugehörigkeit bezieht sich auf die in den Almanachen genannten Regionen, d.h. dass es sich dabei um die jeweiligen historischen Regionen handelt. Mit den sich ständig ändernden Staatsgrenzen wäre es unmöglich gewesen, die Gesellschaften einer heute gültigen Region zuzuweisen. 
Zusätzliche Herausforderungen ergaben sich bei der Vorbereitung der regionalen Bände: Ein großes Hindernis war die Identifizierung der in den Almanachen genannten Städte. Abgesehen von zahlreichen typographischen Fehlern in den Städtenamen war es nicht ungewöhnlich, dass für die Stadt vereinfachte Namen verwendet wurden, mit der Annahme, dass der Leser des Almanachs wissen würde, welche Stadt gemeint ist. Für moderne Anwender der Dokumentation kann dies nicht angenommen werden. Wenn eine eindeutige Identifizierung von Städten in Regionen möglich war, die keine deutschen Namen mehr tragen, wurde der aktuelle Name hinter dem Namen im Haupteintrag platziert und von den Variantenformen auf die in den Almanachen verwendete Form verwiesen. Alle Versuche wurden unternommen, sämtliche Städte zu identifizieren; jedoch mit sehr gebräuchlichen Namen (z.B. Neustadt), ist es möglich, dass eine fehlerhafte Zuordnung gemacht worden ist. ${ }^{38}$

\section{Was kommt noch?}

Nach der Aufbereitung der Inhalte der Almanache in den regionalen und Städte-Bänden wird eine kumulative Ausgabe der Almanach-Dokumentation inklusive der inhaltlichen Erschließung sämtlicher Beiträge in den Almanachen vorbereitet, sodass alle Querhinweise an einer Stelle zu finden sind. Zum Schluss, nachdem möglichst alle zugänglichen lokalen Journale ausgewertet wurden, wird eine Bibliographie mit Inhaltsangaben der lokalen Journale als Ergänzung der Gesamtdokumentation fertiggestellt. Diese Publikationen nehmen, wie bereits erwähnt, einen ganz besonderen Platz in der Dokumentation des Theaters ein: Im Gegensatz zu den Almanachen, ${ }^{39}$ die etwa hinsichtlich Personalstand angeben, wer die Mitglieder eines Unternehmens zu Beginn einer Saison sind ${ }^{40}$ aber nur selten eine umfassende Dokumentation der Bewegung innerhalb des Unternehmens im Laufe einer Saison liefern, dokumentieren die Journale, was während einer Saison tatsächlich geschehen ist, da sie am Ende einer Saison veröffentlicht wurden. ${ }^{41}$ Damit haben die lokalen Theaterjournale eine weit größere

38 Ein Beispiel dafür waren zwei aufeinander folgende Einträge in Deutsches Theater-Lexikon von Adolf Oppenheim und Ernst Gettke für Bautzen. Ein Eintrag war für die Stadt in Sachsen und der andere für Bautzen in Böhmen, letzterer mit einer viel kleineren Bevölkerung. Das Problem ist, dass Bautzen in Sachsen zu verschiedenen Zeiten zu Böhmen gehörte. Und dann gibt es noch das Problem St. Gallen, das nicht nur der Name der bekannten Stadt in der Schweiz, sondern auch einer Gemeinde in Österreich ist.

39 In dieser Hinsicht sind die Almanache den Theaterzetteln ähnlich: Sie geben in der Regel an, was geplant war, nicht jedoch, was tatsächlich geschehen ist.

40 Obwohl es in den Almanachen Hinweise darauf gibt, was in der vorangegangenen Saison passiert ist, sind diese Informationen auch in den besten Fällen lückenhaft. Es sei daran erinnert, dass es eine hohe Mobilität in den Gesellschaften gab, zu der auch die Mobilität der Direktoren gehörte. Ein neuer Direktor einer Gesellschaft wusste im Allgemeinen sehr wenig über die Besonderheiten des Unternehmens seines Vorgängers, und selbst wenn er es wusste, schickte er diese Informationen in der Regel nicht an die Redaktion der Almanache.

41 Dabei ist zu beachten, dass Journale vielen Zwecken dienen können: Viele Bibliotheken katalogisieren sie nicht als Zeitschriften, sondern stellen sie mit den zugehörigen Theaterzetteln zusammen, so dass sie als Register für diese Theaterzettel dienen. Umgekehrt sind Theaterzettelsammlungen selten lückenlos. Der 
Bedeutung für die Dokumentation der tatsächlichen Geschehnisse im Theater.

Mit der Entscheidung der Publikation von eigenen regionalen Bänden wie jenem zu den böhmischen Ländern kann nicht nur ein umfassender Überblick über das Theatergeschehen in der jeweiligen Region gegeben werden, sondern zeigt sich auch die Mobilität von Gesellschaften, Theaterdirektoren und Souffleuren, wenn die kumulierten Materialien aus den regionalen Bänden in einer Gesamtdokumentation zusammengeführt werden.

Ergänzt wird die Gesamtdokumentation durch die Aufnahme von Inhalten, die sich nicht auf theatergeschäftliche Informationen oder auf Einzeltheater beschränken: Mit der Einarbeitung von Informationen zu abgedruckten Stück-Texten, Abbildungen von Personen, Szenen und Spielstätten, Angaben über professionelle Gesellschaften etc. werden weitere Sucheinstiege für spannende Forschungen geschaffen.

\section{Feedback!}

Sie haben Anmerkungen zur oder Desiderate für die Aufbereitung des Quellenmaterials? Sie wissen von Theaterjournalen und -almanachen in Ihrer Einrichtung oder anderswo, die möglicherweise noch nicht berücksichtigt sind? Wir freuen uns auf Ihr Feedback!

\section{Bibliographie}

BLUM, Robert, Karl HERLOSSSOHN und Hermann MARGGRAFF (Hg.). 1839-1846. Allgemeines Theater-Lexikon oder Encyklopädie alles Wissenswerthen für Bühnenkünstler, Dilettanten und Theaterfreunde. Bd. 1-7. Altenburg [u.a.]: Expedition des Theater-Lexikons, 1839-1846.

HANKISS, Elemér und Elisabeth BERCZELI. 1961. A Magyarországon megjelent szinházi zsebkönyvek bibliográfiája. XVIII-XIX. Század = Bibliographie der in Ungarn erschienenen Theaterkalender. XVIII.-XIX. Jahrhundert. Budapest: OSZK, 1961.

HAVLÍČKOVÁ, Margita, Sylva PRACNÁ und Jiří ŠTEFANIDES. 2011-2014. Německojazyčné divadlo na Moravě a ve Slezsku [Deutschsprachiges Theater in Mähren und Schlesien]. 3 Bde. Olomouc: Univerzita Palackého, 2011-2014.

JAKUBCOVÁ, Alena und Matthias J. PERNERSTORFER (Hg.). 2013. Theater in Böhmen, Mähren und Schlesien. Von den Anfängen bis zum Ausgang des 18. Jahrhunderts. Ein Lexikon. Neu bearbeitete, deutschsprachige Ausgabe. Wien: Verlag der Österreichischen Akademie der Wissenschaften, 2013.

OPPENHEIM, Adolf und Ernst GETTKE (Hg.). 1889. Deutsches Theater-Lexikon. Eine Encyklopädie alles Wissenswerthen der Schauspielkunst und Bühnentechnik. Leipzig: Carl Reißner, 1889.

Berliner Forscher Hans-Dieter Meyer, der die Königsberger Theaterzettel in der Sammlung der Akademie der Künste in Berlin katalogisiert hat (siehe http://kultur-in-ostpreussen.de/), hatte viele Lücken in seiner Dokumentation. Mit Hilfe der Königsberger Journale konnte er viele dieser Lücken schließen und auch die Informationen auf den Theaterzetteln überprüfen oder korrigieren. 
PERNERSTORFER, Matthias J. 2018. „Einem hochverehrten Publikum ans Herz gelegt ...“. Bibliographische \& inhaltliche Erschließung, Digitalisierung und Präsentation von Theaterjournalen und -almanachen. In Digitalisierung in Bibliotheken. Viel mehr als nur Bücher scannen, hg. von Gregor Neuböck. Berlin/Boston: De Gruyter Saur, 2018: 147-172.

PERNERSTORFER, Matthias J. und Andrea GRUBER. 2015. Bibliografische Forschung als Grundlage für Digitalisierungsprojekte. Oder: ,Theaterpublizistik 1750-1918 digital‘. Ein Projekt von Paul S. Ulrich und dem Don Juan-Archiv Wien. AKMB-News 21 (2015): H. 1: 18-21.

SACHSE, Carl Albrecht (Hg.). 1853-1872. Statistisches Handbuch für Bühnenvorstände, Bühnenkünstler und Bühnenfreunde. Hamburg, 1853-1855; Wien, 1865-1872.

ULRICH, Paul S. 1997. Biographisches Verzeichnis für Theater, Tanz und Musik. Fundstellennachweis aus deutschsprachigen Nachschlagewerken und Jahrbüchern. 2 Bde. Berlin: Berliner Wissenschafts-Verlag, 1997.

ULRICH, Paul S. 2018. Wiener Theater (1752-1918). Dokumentation zu Topographie und Repertoire anhand von universalen Theateralmanachen und lokalen Theaterjournalen. Mit einem Überblick zu Zeitungen mit Theaterreferaten und deren Referenten. Wien: Hollitzer Verlag, 2018. (= Topographie und Repertoire des Theaters 1)

ULRICH, Paul S. 2019. Almanache als Quellen zur Dokumentation des deutschsprachigen Theaters bis 1918. AKMB-News 25 (2019): H. 1: 38-43.

\section{Paul S. Ulrich}

Bamberger Str. 53, 10777 Berlin, Deutschland

psulrich@gmx.de

Paul S. Ulrich, 1976 bis 2009 Bibliothekar in der Zentral- und Landesbibliothek Berlin (bis 1994 Amerika Gedenkbibliothek), seit 2005 Vorsitzender der Gesellschaft für Theatergeschichte, Schatzmeister und Mitherausgeber der Veröffentlichungen von Thalia Germanica. Wichtigste Veröffentlichungen: Biographisches Verzeichnis zu Theater, Tanz und Musik (1997), Das Jahr 1848. Kultur in Berlin im Spiegel der Vossischen Zeitung (2008) und Wiener Theater (17521918) (2018). Zahlreiche Aufsätze über Theater-Almanache und -Journale (s. https://www. theatergeschichte.org/mitgliedschaft/publikationslisten-der-mitglieder/paul-s-ulrich/) 